\title{
Magnetic ultrathin films and multilayers: growth and characterization
}

\author{
J.-P. Deville ${ }^{*}$, A. Barbier ${ }^{3}$, C. Mocuta ${ }^{3}$, G. Renaud 3 , Y. Samson ${ }^{3}$, H. Magnan ${ }^{4,5}$, P. Le Fèvre , \\ D. Chandesris ${ }^{4}$, P. Panissod ${ }^{2}$ and C. Mény ${ }^{1}$ \\ ${ }^{1}$ IPCMS, Groupe Surfaces Interfaces, UMR 7504, CNRS-ULP, 23, rue du Loess, 67037 Strasbourg Cedex, France \\ ${ }^{2}$ IPCMS, Groupe d'Études des Matériaux métalliques, UMR 7504, CNRS-ULP, 23, rue du Loess, 67037 Strasbourg Cedex, France \\ ${ }^{3}$ CEA/Grenoble, Département de Recherche Fondamentale sur la Matière Condensée, SP2M, 17, rue des Martyrs, \\ 38054 Grenoble Cedex 9, France \\ ${ }^{4}$ LURE, Université Paris-Sud, 91898 Orsay Cedex, France \\ ${ }^{5}$ CEA, SRSIM, 91191 Gif-sur-Yvette Cedex, France
}

This report intends to address the results obtained in the recent years about the growth and structure of magnetic artificial architectures such as ultrathin films, sandwiches, multilayers and superlattices.

\section{Magnetic metallic multilayers and new devices}

The development of new devices based on magnetic metallic multilayers (MMM) has known a tremendous expansion during the last decade and it led to a new field of research and development which can be called "magnetoelectronics". Among these new applications, one can cite sensors for linear displacements [1], contactless potentiometers [2], rotation, angular and position sensing [3], measurements of weak fields [4], microcompass [5], read heads [6], magnetic random access memories (MRAM's) based either on tunneling magnetoresistance (TMR) [7], on exchange-biased spin-valves [8] or on pseudo-spin-valve structures [9], spin transistors. A recent review [10] describes quite extensively all these applications.

The theory of thin film magnetism was much developed along the fifties and sixties, particularly by Néel who discussed several effects such as the surface magnetic anisotropy [11], the magneto-coupling [12] and the interfacial coupling [13] but the experimental developments are much more recent and followed somewhat lately the ways of semiconductor technologies such as molecular beam epitaxy (MBE), building of complex structures, patterning... These developments have given rise to new magnetic effects based on such properties known as uniaxial anisotropy or magnetoresistance. One can cite the perpendicular magnetic anisotropy in layered structures $[14,15]$, the indirect coupling exchange [16,17], the giant magnetoresistance $[18,19]$, the large oscillation periods of antiferromagnetic coupling [20] soon followed by the discovery of the short oscil- lation periods [21], the spin-valve effect [22], the magnetic tunnelling effect [23]. More recently, colossal magnetoresistance (CMR) effects have been shown in materials having the perovskite structure [24]. Although this is not a property directly linked to low-dimensionality effects, it is worth noting it since nowadays a large development of these materials occurs in magnetic thin film technology.

These experimental discoveries have of course induced many new theoretical insights and one can find a good description of the state of art in Ref. [25].

Most of these magnetic properties depend on parameters which are directly related to the morphology, the crystallography and the chemistry of the system, i.e. the way the various layers are grown and the atoms more or less ordered in the artificial structure. Unfortunately, the role of these parameters is hardly predictible. For example, in $\mathrm{Fe} / \mathrm{Cr}$ multilayers interfacial roughness increases the GMR [26,27] whereas, on the contrary, to favor the increase of GMR in $\mathrm{Co} / \mathrm{Cu}$ systems one generally needs abrupt interfaces $[28,29]$. So, a full characterization of the system will be necessary to correct the growth parameters and optimize the properties. Moreover, to understand these magnetic properties and to obtain convincing correlations between them and the crystallographic structure, one often needs to develop model systems based on simpler configurations such as ultrathin films (thicknesses lower than $1 \mathrm{~nm}$ ) deposited on single crystals or sandwiches.

In this paper we shall restrict ourselves to the structural analysis on a microscopic scale and we shall not describe the methods for measuring the magnetic properties. Generally, if one wants to perform such measurements in situ on samples like ultrathin films or sandwiches one has to use physical methods such as (Surface) Magnetic Optical Kerr Effect [(S)MOKE)] or magnetic dichroism, keeping in mind that these techniques do not offer easy quantitative measurements. For multilayers, conventional methods will be used: Vibrating Sample Magnetometer (VSM) or Alternating Gradient Force Magnetometry (AGFM). 


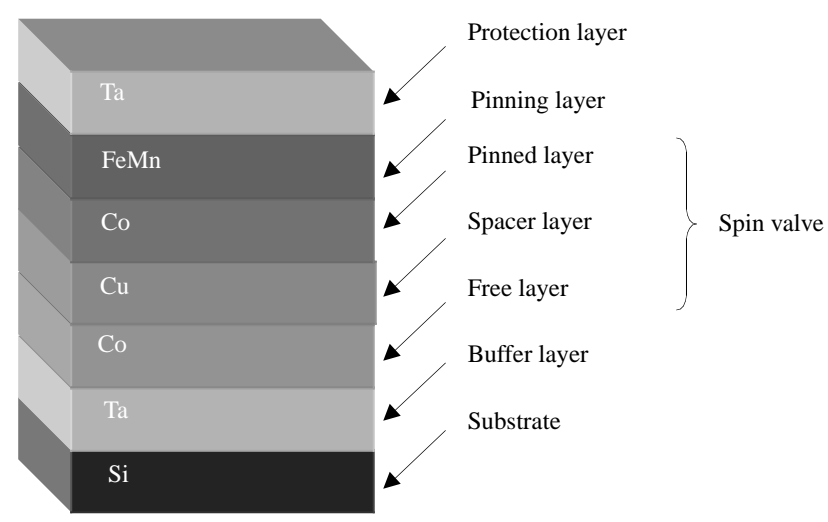

Figure 1. The various constitutive layers of a typical spin valve [52].

\section{Growth and morphology}

A magnetic multilayer consists in a stack of various metallic or oxide layers which are deposited alternatively on a substrate, generally silicon, magnesium oxide or sapphire. A thick $(\cong 10-100 \mathrm{~nm})$ buffer layer is often deposited on the substrate to act as a sead layer. Then in the "active" architecture the thickness of each layer is in the nanometer range. Figure 1 shows a typical structure of a spin valve.

\section{Morphology}

The layer morphology depends on the growth methods, which are either physical deposition such as molecular beam epitaxy (MBE), sputtering and pulsed laser deposition (PLD) or chemical processes such as electrochemical deposition. There is always a competition between thermodynamics and kinetics parameters [30]. Thermodynamics gives four main criteria, which are temperature dependent:

(i) the elements having the smaller surface tension will wet those having a higher one,

(ii) the elements having the larger atomic radius will tend to segregate toward the surface,

(iii) if there is a miscibility (even rather small) between the elements intermixing is expected,

(iv) if there is a tendency to chemical order in the bulk phases, surface alloys can appear inducing defects such as antiphase boundaries, strain and unexpected magnetic properties.

The main parameter for kinetics will be the surface diffusion coefficient of the atoms which is also temperature dependent. If this coefficient is large enough, a smooth surface is expected. However, structural defects present at the surface (steps, superstructures, islands already grown) will act as traps for the incoming atoms and modify the growth conditions. A wise analysis of these often conflicting parameters will allow an engineering of the thin films. For example, one can use surfactants, i.e. low surface tension elements which are predeposited and float all along the growth. A good example of the role of $\mathrm{Pb}$ on the growth and magnetic properties of $\mathrm{Co} / \mathrm{Cu}$ superlattices has been given, among others, by Camarero et al. [31]. The surfactant induces a layer-by-layer growth of cobalt and delays the face-centered cubic (fcc) toward hexagonal (hcp) phase transition. In this case, the easy axis of magnetization remains perpendicular to the plane. $\mathrm{Pb}$ also suppresses structural defects such as twins and this allows to observe a perfect antiferromagnetic (AF) coupling between the Co layers across the copper spacer. This is of course important for the understanding of the mechanisms involved in micromagnetism. There are some drawbacks since the surfactant can be incorporated as an impurity in the structure, inducing modifications of the magnetic properties. For the same system, $\mathrm{Co} / \mathrm{Cu}$, one can try to act on the thermodynamics side using another surfactant such as oxygen that will be easier to eliminate [32]. One can either act on the kinetics side and adapt the surface diffusion coefficient, using plasma laser deposition instead of thermal evaporation [33].

\section{Growth modes}

There are three growth modes at thermodynamical equilibrium:

(i) Frank-van der Merwe which corresponds to a layer-by layer growth (full wetting of the substrate by the adsorbate);

(ii) Stranski-Krastanov in which the development of islands occurs after the growth of one or two wetting layers;

(iii) Volmer-Weber which is the unwetting situation, characterized by the growth of islands.

Of course, the systems are practically never at equilibrium during the growth and one often departs from these growth modes: layers can grow simultaneously, interdiffusion may occur...

The growth modes can be determined in situ either by scanning tunnelling microscopy (STM) as seen in the chapter "From random to self-organized..." in which figure 3 illustrates the growth of a 2D array of cobalt clusters on top of a $\mathrm{Au}(111)$ surface reconstruction [34], by reflexion high energy electron diffraction (RHEED) in which one monitors the intensity of the specular reflexion [21] or by Auger electron spectroscopy (AES). In this latter case, one plots continuously during the growth two (or more if possible) Auger signals which are characteristic respectively of the substrate and of the adsorbate. Applying simple relations between the signal intensities, their energy and the coverage of each atomic layer, then assuming various shapes for the islands allow one to modelize the Auger signals and to decide which growth mode occurs. Figure 2 shows such calculations for the deposition of platinum on a cobalt single crystal surface [35], describing a good layer-by-layer growth ("Frankvan der Merwe"). 


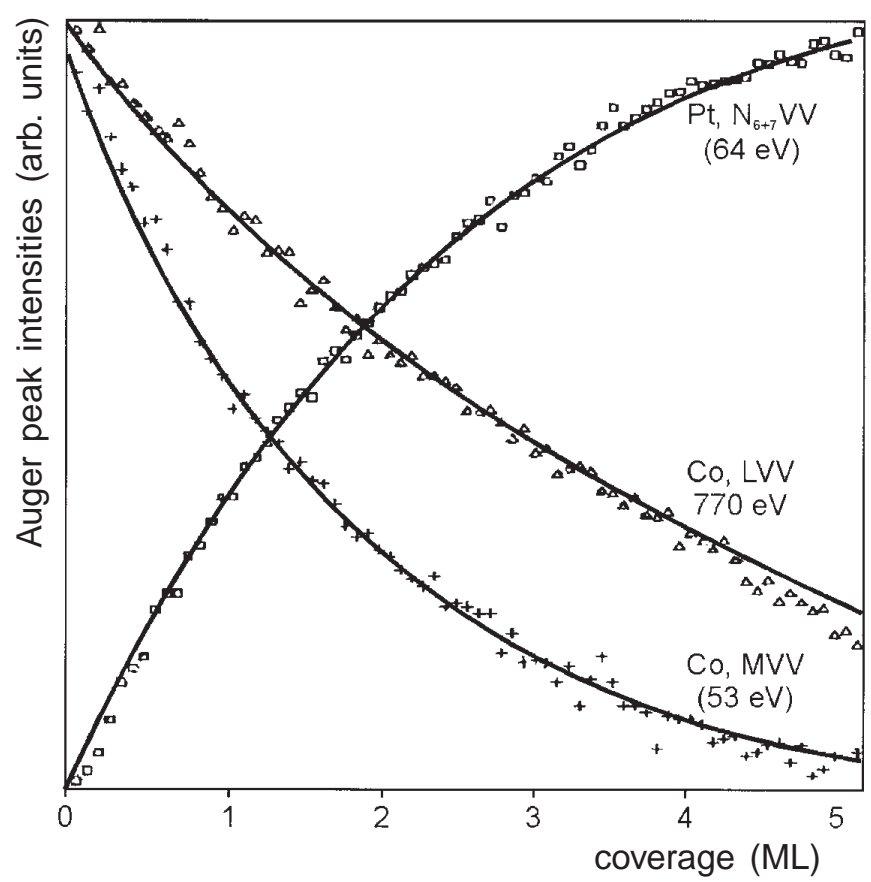

Figure 2. Study of the deposition of platinum on cobalt. Pt and Co Auger signals versus platinum coverage. The continous lines represent the best fit for a layer-by-layer growth mode.

These three methods (STM, RHEED, AES) are very surface sensitive and probe only the topmost layers. They do not give informations about the evolution of the layers which are progressively buried. They do not give either precise informations about the local atomic structure: for STM, the interpretation of the chemical contrast between the various atoms is far from being simple, AES is not a method able to give structural informations and in the case of RHEED the dynamical effects prevent one to extract an exact crystallographic structure.

\section{Growth and structure}

STM and low-energy-electron-diffraction (LEED) are ideal structural methods to investigate the first stages of growth, in the real space for the first technique, in the reciprocal space for the second one. For example, LEED, combined with AES is able to identify complex structures such as the $\mathrm{bcc/fcc}$ or bcc/fcc interfaces [36] and to assess the role of epitaxial stress [37]. However as STM, it is restricted to the very first layers and sometimes not precise enough. None of these methods are suitable for ex situ analysis.

One will have to appeal to less surface limited and/or more precise methods such as grazing incidence X-ray diffraction, Extended X-ray Absorption Fine Structure (EXAFS), Nuclear Magnetic Resonance (NMR), high resolution transmission electron microscopy (HRTEM) which will be also able to probe buried interfaces. Four examples will be given in the following paragraphs to illustrate these methods.

\section{Structure of the Co/NiO(111) interface}

The $\mathrm{Co} / \mathrm{NiO}(111)$ system is promising in the framework of devices based on the spin-valve geometry. $\mathrm{NiO}(111)$ is an antiferromagnetic polar oxide insulator highly resistant to oxidation and with a high Néel temperature (523 K). It is a good candidate for providing the magnetic pinning mandatory in spin-valve structures.

This study was carried out on the French CRG-IF X-ray beamline at ESRF (European Synchrotron Radiation Facility, Grenoble, France), by Grazing Incidence X-ray Diffraction (GIXD) because this relatively recent technique [38] is insensitive to the charge built-up. In an earlier work [39], it has been shown that the $\mathrm{p}(2 \times 2)$ stabilized (111) surface of the $\mathrm{NiO}(111)$ single crystal exists. Here, the $\mathrm{Co} / \mathrm{NiO}(111)$ interface was investigated during its formation, starting from the very early stages of deposition up to fairly thick films ( $\sim 20 \mathrm{~nm}$ of Co) with different substrate temperatures: room temperature (RT) and high temperature (HT) (703 K and $773 \mathrm{~K}$ ).

The NiO(111) surface was indexed using a hexagonal surface unit cell. From the symmetry of the system, hcp, fcc (the stacking which continues the $\mathrm{NiO}$ one) and twined-fcc Co (rotated by $60^{\circ}$ ) could be expected. In-plane scans along the $[h, h, 0]$ direction (similar to a point by point description of a low energy electron diffraction pattern) passing through the Co Bragg peak common to all these stacking show a great difference between the two situations. For the RT deposit, the signal of crystalline Co is found to appear only after $\sim 1 \mathrm{~nm}$ of Co deposition, whereas for the HT deposit, scattering is already found at the very early stages of growth. Moreover, for $20 \mathrm{~nm}$ thick films, the intensity is 200 times larger for HT deposits. Thus the RT deposit is poorly ordered whereas the HT deposit is epitaxial and well ordered.

Out of plane scans along the $[1,0, \ell]_{\mathrm{Co}}$ rod allow the distinction between different Co stacking because they cross successive Bragg peaks of hcp, fcc and twined-fcc stacking (Fig. 3), this information is not accessible through other techniques. At the end of the RT deposit, the $[1,0, \ell]_{\text {Co }}$ rod shows no peaks at the expected Co positions. It is only after recrystallization (by annealing the film 45 minutes at $973 \mathrm{~K}$ ) that fcc, twined-fcc and hcp Co peaks appear. Integrated and corrected intensities of the peaks make this technique fully quantitative and show the presence of all stacking in almost equal amounts in the Co layer. For HT deposits, the intensity of all Co peaks increases with Co thickness. The fcc stacking is strongly dominant for all thicknesses, but residual contributions of all other stacking are present for the deposit at $703 \mathrm{~K}$. At $773 \mathrm{~K}$, no hep Co is found and 98-99 \% of the $20 \mathrm{~nm}$ thick Co film is fcc. Thus the metastable Co fcc phase has been stabilized for a very large deposited thickness. 


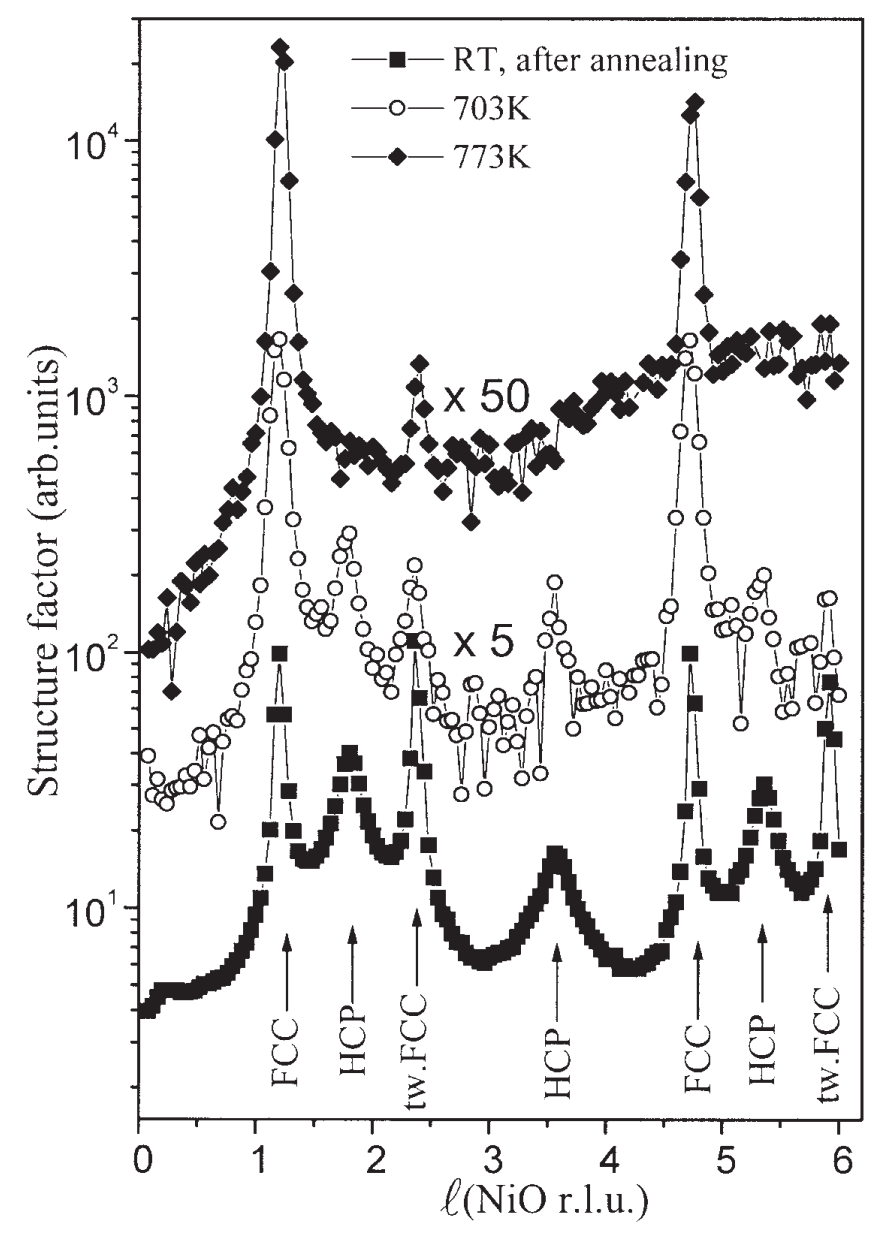

Figure 3. $[1,0, \ell]_{\mathrm{Co}}$ rod for the RT, $703 \mathrm{~K}$ and $773 \mathrm{~K}$ deposits. The scans were vertically shifted $(x 5: 703 \mathrm{~K}$ and $x 50: 773 \mathrm{~K})$ for the sake of clarity. Positions where peaks from fcc, hcp and twinned fcc stacking are expected [in the reciprocal lattice units of $\mathrm{NiO}$ (r.l.u.)] are indicated by arrows.

To summarize the growth mode of $\mathrm{Co}$ on $\mathrm{NiO}(111)$ is of the Volmer-Weber type at both temperatures, but the internal crystallographic structure of the clusters can be varied from nearly amorphous to perfectly epitaxial with fcc Co continuing the $\mathrm{NiO}(111)$ lattice although the lattice misfit is about $17 \%$.

\section{EXAFS a method for thin film, interfaces and multilayer structural characterization}

When deposited as ultrathin films or multilayers, magnetic metals are often in a metastable crystallographic structure. Stress induced by the epitaxy on a substrate leads to non standard structures, showing distorsions due to the size mismatch between the adsorbed atoms and those of the substrate. The presence of these new phases joined to interfaces and low-dimensionality effects modifies the electronic structure of the thin films and induces original magnetic properties. EXAFS (Extended X-ray Absorption Fine Structure) is

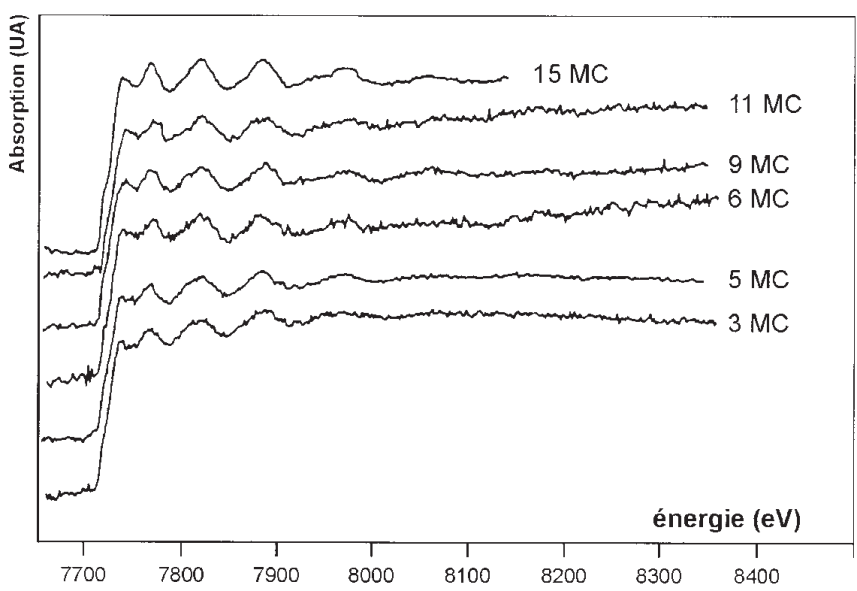

Figure 4a. Deposition of cobalt on $\operatorname{Cr}(001)$. Experimental SEXAFS spectra at the Co $\mathrm{K}$ edge as a function of Co coverage.
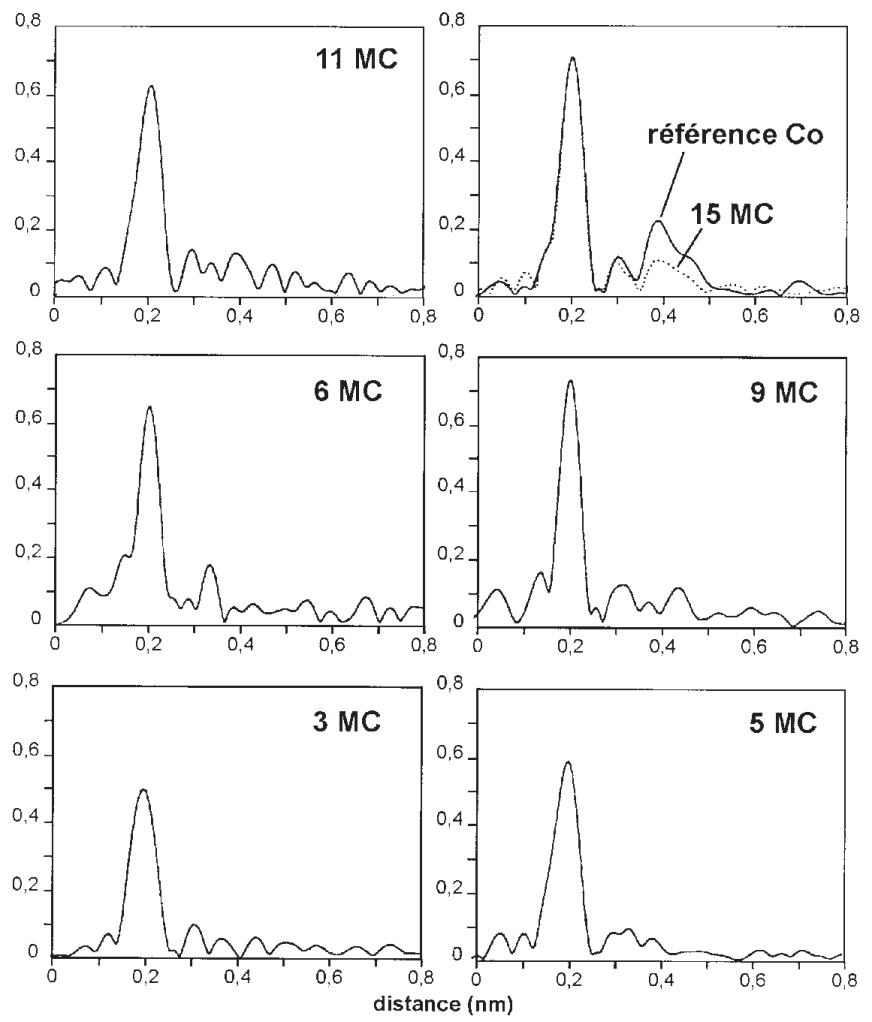

Figure $4 b$. Fourier tranforms of the spectra in figure $3 a$.

a powerful technique to investigate these structure modifications. It consists in a measurement of the X-ray absorption coefficient of a material as a function of the incident photon energy. In a solid, oscillations of this absorption coefficient are observed after each absorption edge: they are characteristic of the local crystal order around the absorbing 
atom [40]. Figure 4 shows typical EXAFS spectra and the corresponding Fourier transforms obtained for cobalt films with various thicknesses deposited on $\mathrm{Cr}(001)$ single crystal surfaces [41].

EXAFS has three main advantages:

(i) it is element selective: if one scans an absorption edge of a given element present in the material, one probes only the environment of this particular atom. This chemical selectivity allows also the study of buried interfaces or of the various constitutive layers of a superlattice;

(ii) it addresses local order: unlike diffraction, EXAFS does not require any long range order and can be applied to amorphous materials;

(iii) it is sensitive to structural distortions if ones takes advantage of the linear polarization of the synchrotron radiation source [42]. By varying the incidence angle of the X-ray beam, one can favor the contribution of given crystalline axes to EXAFS oscillations.

Numerous studies on magnetic metallic multilayers, sandwiches or ultrathin films have been performed and some of them are described below. If one is more interested in EXAFS, the reading of Ref. [43] can be useful.

When deposited onto a $\mathrm{Cu}(001)$ substrate, iron ultrathin films are stabilized in a fcc structure. EXAFS studies have shown that the preparation modes of the samples (deposition temperature, annealings, or even a coverage of the iron film by copper atoms) induce large variations of the iron crystal structure. In particular, a perpendicular magnetization to the layers has been correlated with an anisotropic disordered structure of the iron deposits [44].

The $\mathrm{Co} / \mathrm{Cu}(001)$ system has been among the first systems where bidimensional magnetism was studied. EXAFS has demonstrated that cobalt was in a fct (face centered tetragonal structure). The parameter parallel to the $\mathrm{Co} / \mathrm{Cu}$ interface is equal to that of copper and perpendicularly it is somewhat smaller. The epitaxy of cobalt on copper induces a lateral expansion of the cobalt lattice, leading to a longitunal compression which is very well described within a simple elastic model [42]. Using a modelization programs within a multiple diffusion formalism [45] has also allowed to describe the growth of cobalt on $\mathrm{Cu}(111)$ and to determine the stacking of the compact planes [46].

In multilayers, EXAFS evidenced structural modifications of copper (bcc or fcc) in iron-copper multilayers, related to the copper thickness [47].

\section{NMR analysis of multilayered or granular magnetic nanocomposites}

In magnetic materials, the large spontaneous field acting on the nuclei, and consequently their resonance frequency, depends strongly on their local environment (number, distance and nature of the neighbours). Therefore NMR can be very useful to identify the various magnetic phases present in composite materials, to study their local atomic structure and to reveal the topology of their interfaces. This gives a local insight on the structure in the direct space, which complements the structural information from standard X-ray diffraction and electronic microscopy observations. In terms of probed distances and element selectivity NMR can be compared to the EXAFS technique: while, contrary to EXAFS, NMR only yields qualitative information about inter-atomic distances, it provides much more detailed information about local chemical configurations. Actually the NMR spectrum reflects the occurrence probability distribution of the atomic configurations in a sample (each different chemical co-ordination giving rise to a characteristic line in the spectrum). The archetype of such a distribution is the one observed in binary solid solutions $\mathrm{A}_{1-x} \mathrm{~B}_{x}$ : starting from a single line for the pure magnetic element $\mathrm{A}$, the spectrum develops nearly equally spaced satellite lines when $\mathrm{A}$ is alloyed to $\mathrm{B}$. The successive satellites (see Fig. 5a) correspond to nuclei with $1,2,3$, etc. $\mathrm{B}$ atoms substituted for $\mathrm{A}$ in their co-ordination shell. In a good solid solution, the configuration probabilities, and thus the spectral shape, follow a binomial distribution. Deviation from this law indicate preferential substitution due to chemical ordering or segregation. At interfaces of artificially layered systems like those prepared by sequential deposition (multilayers) each atom is supposed to have a well defined chemical co-ordination and, therefore, the interfacial atoms show up in the spectrum as a well resolved line separated from the main one (that of the atoms inside the layer). Figure $5 \mathrm{~b}$ shows such a case for a Co layer deposited on the (111) face of a $\mathrm{Cu}$ single crystal: the spectrum shows, indeed, that the interface spectrum consists essentially in one line around $170 \mathrm{MHz}$ corresponding to Co nuclei with $3 \mathrm{Cu}$ atoms among their 12 nearest neighbours; the Co layer is still defective, though, as it shows Co in its two stable phases, the low temperature one, hexagonal compact (majority) at $226 \mathrm{MHz}$ and the high temperature one, face centered cubic at $217 \mathrm{MHz}$. This is an example of how the smaller atomic distances in hcp Co induce a larger resonance frequency with respect to fcc Co. Note that the situation is reversed for the satellite: the 3 non magnetic $\mathrm{Cu}$ neighbours depress more the field on Co nuclei at the hcp $\mathrm{Co}-\mathrm{fcc} \mathrm{Cu}$ interface than at the fcc $\mathrm{Co}-\mathrm{fcc} \mathrm{Cu}$ interface. The case of a much less perfect $\mathrm{Co} / \mathrm{Cu}$ multilayer is shown in figure 1c: here the interface Co nuclei are embedded in a large variety of chemical co-ordinations with 1 to 4 and more $\mathrm{Cu}$ neighbours, which is due to an interdiffusion over several atomic layers. Note the very different shape of the spectrum tail below the main line as compared to that of the homogeneous alloy: this is due to the large concentration gradient at the interface. Note also that a trace of perfect interface is still visible as a slightly prominent satellite at $170 \mathrm{MHz}$. From the relative intensities of the lines, the fraction of nuclei at the interface is estimated, which shows immediately the amount of admixture at the interface. A more detailed information is gained by modelling the spectral shape: concentration profiles, densities of step defects, sizes of grains or islands can be evaluated thus characterising the interface roughness at atomic distance scale. 


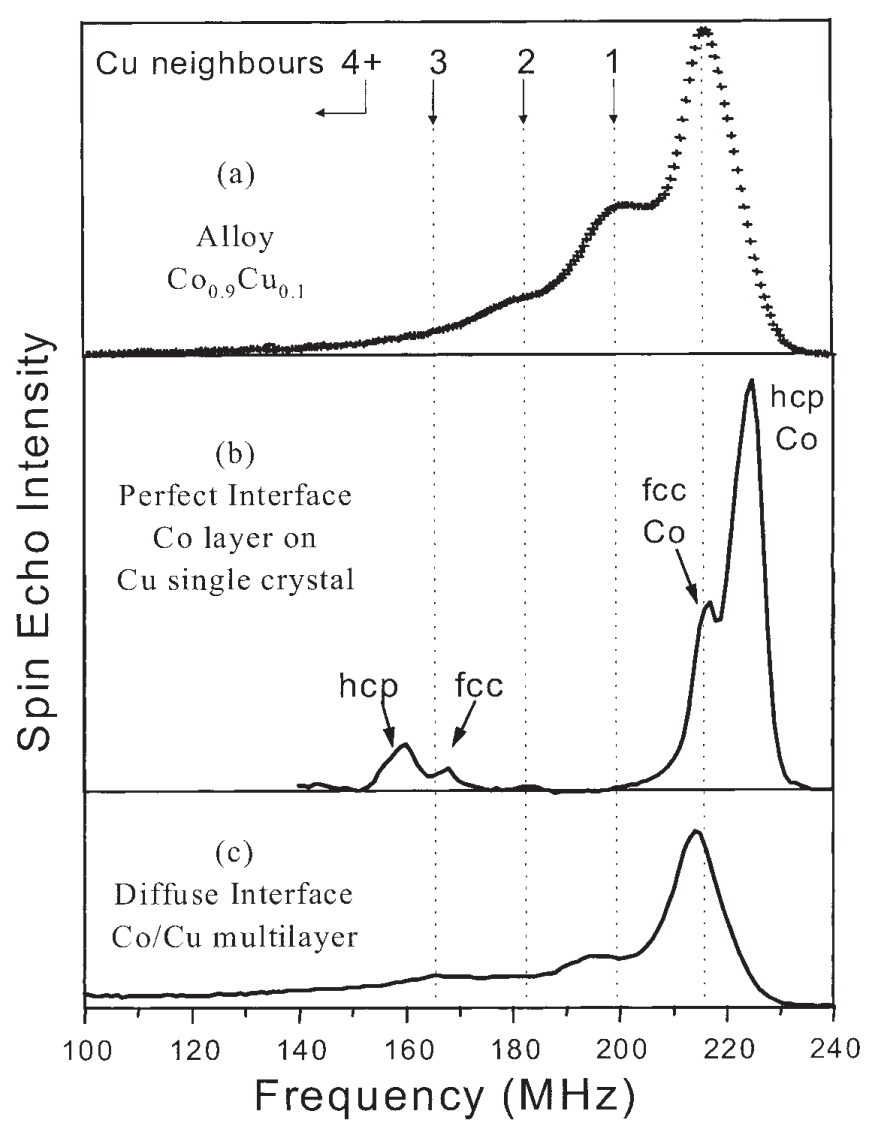

Figure 5. NMR spectra of $\mathrm{Co} / \mathrm{Cu}$ alloys, perfect interface and multilayers.

The technique applies to granular systems as well. Figure 6 shows the spectra observed for granules of cobalt in silica as obtained by reduction of a sol-gel of $\mathrm{Co}+\mathrm{Si}$ hydroxides. At temperature below $850{ }^{\circ} \mathrm{C}$ only small granules of fcc Co are formed, which contain a lot of hcp stacking faults evidenced by the successive shoulders on the high frequency side of the spectrum. The position of the main peak is here $223 \mathrm{MHz}$ because the demagnetising field adds $6 \mathrm{MHz}$ to the internal field of single domain magnetic spheres (diameter $<30 \mathrm{~nm}$ ); actually the thermal evolution of the spectrum between $4.2 \mathrm{~K}$ and $300 \mathrm{~K}$ sets the diameter of these spheres between 1 and $3 \mathrm{~nm}$. At higher reduction temperatures these small clusters are better and better crystallised due to the effect of annealing but their volume fraction does not change. Correspondingly, above $800{ }^{\circ} \mathrm{C}$, a new family of much larger fcc Co clusters $(\mathrm{d}>30 \mathrm{~nm})$ are formed, as evidenced by their regular fcc Co line at $217 \mathrm{MHz}$. This second family is supposed to arise from the reduction of large Co silicide grains that form at intermediate temperatures as evidenced by transmission electron microscopy. Because of this two step reduction process, the final distribution of Co cluster sizes in the sample is clearly bimodal. If interested to learn more about NMR in magnetic nanosystems, one can read Ref. [48].

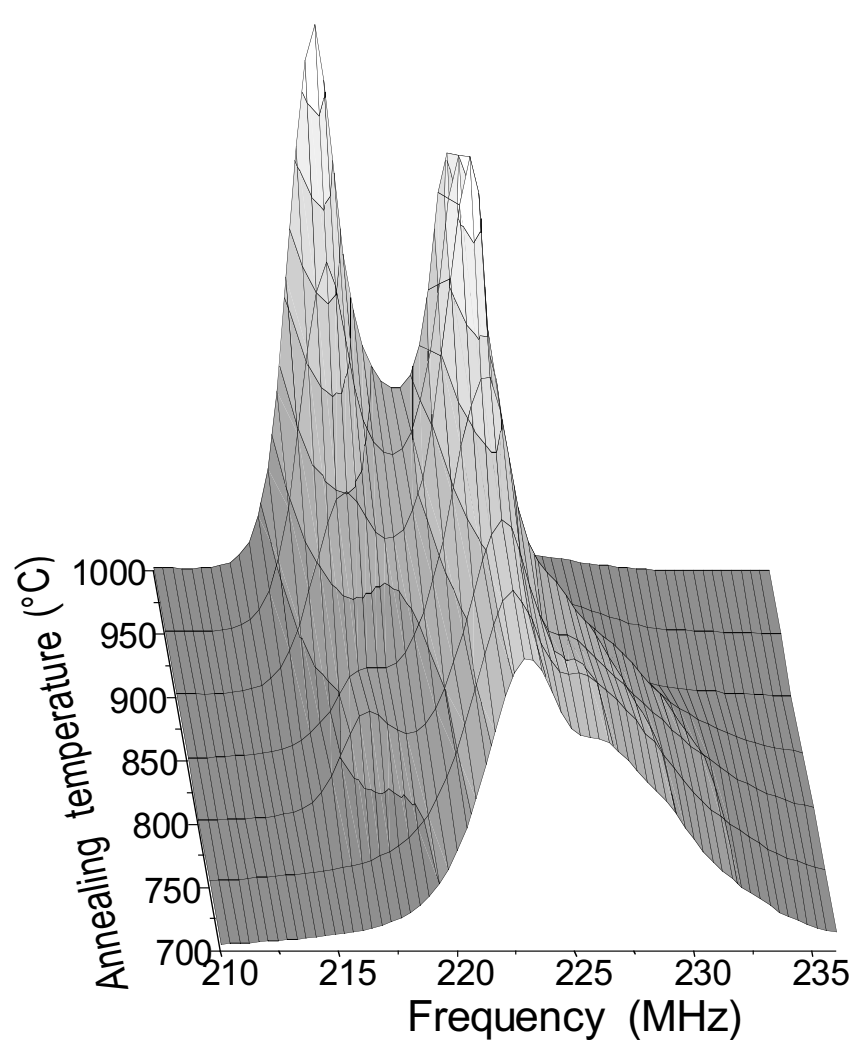

Figure 6. Variation of the RMN spectrum as a function of annealing temperature for Co granules in silica.

\section{High resolution transmission electron microscopy of interfaces in spin valves}

Transmission electron microscopy is a powerful technique for investigating thin film materials. It allows to obtain images either as dark or bright field micrographs when operating in the normal mode or as representations of the arrangements of atom columns when using the high resolution mode. An identification of the crystalline phases is also possible if using the microdiffraction mode. There are of course some drawbacks:

(i) the thickness of the samples must be less than a tenth of a micrometer for conventional microscopy and around $10 \mathrm{~nm}$ for high resolution. It is thus mandatory to prepare the samples before their observation through a thinning procedure. This is a destructive method and observing samples in situ will not be possible.

(ii) the interpretation of the diagrams in the high resolution mode needs careful simulations to take into account successively the interaction of the partially coherent incident electron wave with the crystal potential, its propagation in the crystal and the transfer of the wave front in the image plane of the microscope. 


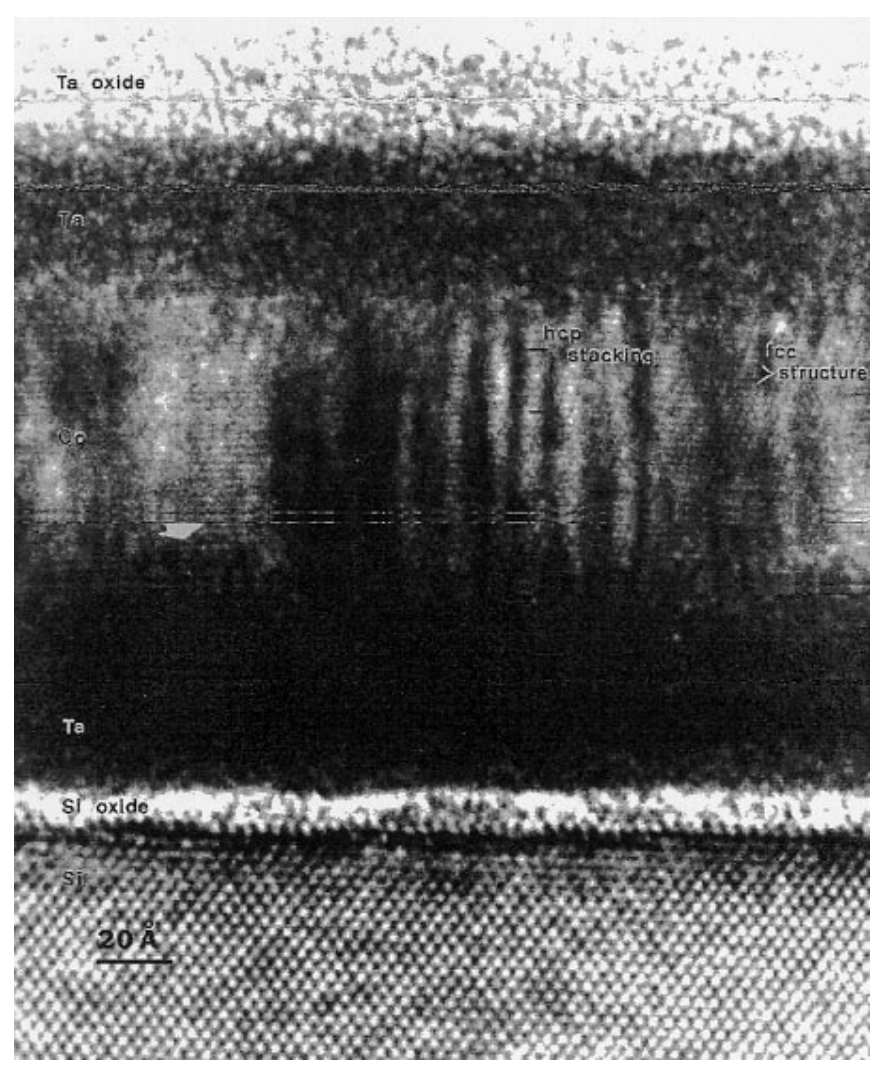

Figure 7. High resolution image of a Si(001)/Ta $5 \mathrm{~nm} / \mathrm{Co} 8 \mathrm{~nm} / \mathrm{Ta}$ $5 \mathrm{~nm}$ sandwich obtained along Si [110].

(iii) the image contrast between atoms having atomic numbers close together is weak. Imaging cross sections of magnetic multilayers the constitutive elements of which are generally from the first series of transition metals will be difficult.

Spin-valves [49], an example of which is shown in figure 1 , are magnetoresistive systems in which a non magnetic layer $(\mathrm{Cu})$ is sandwiched between two ferromagnetic (FM) layers $\left(\mathrm{Co}_{1}\right.$ and $\left.\mathrm{Co}_{2}\right)$ and topped by an antiferromagnetic (AF) layer (FeMn). They present a large magnetoresistance for weak magnetic fields and can be applied in read heads. Actually the architecture is much more complicated as can be seen in figure 1. In such systems, the magnetoresistance depends on the angle between the magnetization vectors of the two ferromagnetic layers. The magnetization of the $\mathrm{Co}_{2}$ layer is trapped by the AF layer by exchange anisotropy. Spin dependent diffusion can explain the magnetoresistance but it can depend either on interface or bulk effects. Figure 7 shows a micrograph obtained on a simplified structure $[50,51]$ obtained by sputter deposition at room temperature. One can see the columnar growth of cobalt on the tantalum buffer layer: the column orientation is parallel to the growth direction. One can also note stacking faults in the cobalt layer since cobalt can be either hcp or fcc.
Similar structures has been observed in Co-Ag layers obtained by MBE deposition at various temperature [52] with different $\mathrm{Ag}$ concentrations. In this case, electron microscopy has been used to find the best growth conditions to obtain well characterized granular $\mathrm{Co}-\mathrm{Ag}$ films having good magnetoresistive properties.

\section{Conclusion}

The number of applications of magnetic metallic multilayers is growing very fastly and the analysis of the growth and structure of their interfaces can be made with several methods. It should be emphasized that one cannot rely on a single one: many different approaches must be performed to obtain convincing and reliable results. One has to study the long range and the local orders, the chemistry at the interfaces and, of course, obtain correlations with the magnetic properties.

\section{References}

1. Miller, M. M.; Prinz, G. A.; Lubitz, P.; Hoines, L.; Krebs, J. J.; Cheng, S. F.; Parsons, F. G. J. Appl. Phys. 1997, 81, 42844286.

2. Clemens,W.; van den Berg, H. A. M.; Rupp, G.; Schelter, W.; Vieth, M.; Wecker, J. J. Appl. Phys. 1997, 81, 4310-4312.

3. Suzuki, M.; Ohwaki, T.; Taga, Y. Thin Solid Films 1997, 304, 333-338.

4. Schuhl; A.; Nguyen Van Dau, F.; Childress, J. R. Appl. Phys. Lett. 1995, 66, 2751-2753.

5. Montaigne, F.; Schuhl, A.; Nguyen Van Dau, F.; Encinas, A. Sensors and Actuators 1999, in press.

6. Tsang, C.H.; Fontana Jr, R. E.; Lin, T.; Heim, D. E.; Gurney, B. A.; Williams, M. L. IBM J. Res. Develop. 1998, 42, 103116.

7. Gallagher, W. J.; Kaufman, J. H.; Parkin, S. S. P.; Scheuerlein, R. E. US Patent 564343, 1997.

8. Kools, J. C. S. IEEE Trans. Magn. 1996, 32, 3165-3184.

9. Tehrani, S.; Chen, E.; Durlam, M.; DeHerrera, M.; Slaughter, J. M.; Shi, J.; Kerszykowski, G. J. Appl. Phys. 1999, 85, 58225827.

10. Prinz, G. A. J. Magn. Magn Mater. 1999, 200, 57-68.

11. Néel, L. J. Phys. Rad. 1954, 15, 225-239.

12. Néel, L. C. R. Acad. Sci. 1962, 255, 1676-1681.

13. Néel, L. Ann. Phys. 1967, 2, 61-80.

14. Carcia, P. F.; Meinhaldt, A. D.; Suna, A. Appl. Phys. Lett. 1985, 47, 178-180.

15. den Broeder, F. J. A.; Hoving, W.; Bloemen, P. J. H. J. Magn. Magn. Mater. 1991, 121, 562-570.

16. Grünberg, P.; Schreiber, R.; Pang, Y.; Brodsky, M. B.; Sowers, H. Phys. Rev. Lett., 1986, 57, 2442-2445.

17. Carbone, C.; Alvarado, S. F. Phys. Rev. B 1987, 36, 24432445.

18. Baibich, M. N.; Broto, J. M.; Fert, A.; Nguyen Van Dau, F.; Petroff, F.; Etienne, P.; Creuzet, G.; Friederich, A.; Chazelas, J. Phys. Rev. Lett. 1988, 61, 2472-2475. 
19. Binasch, G.; Grünberg, P.; Saurenbach, F.; Zinn, W. Phys. Rev. $B$ 1989, 39, 4828-4850.

20. Parkin, S. S. P.; More, N.; Roche, K. P. Phys. Rev. Lett. 1990 64, 2304-2307.

21. Unguris, J.; Celotta, R. J.; Pierce, D. T. Phys. Rev. Lett. 1991, 67, 140-143.

22. Dieny, B.; Speriosu, V. S.; Parkin, S. S. P.; Gurney, B. A.; Wilhoit, D. R.; Mauri, D. Phys. Rev. B 1991, 43, 1297-1300.

23. Miyazaki, T.; Tezuka, N. J. Magn. Magn. Mater. 1995, 139, L231-L235.

24. van Helmolt, R.; Wecker, J.; Holzapfel, B.; Schultz, L.; Samwer, K. Phys. Rev. Lett. 1993, 71, 2331-2335.

25. Dreyssé, H.; Demangeat, C. Surface Science Reports 1997, 231, 1-57.

26. Petroff, F.; Barthélémy, A.; Hamzic, A.; Fert, A.; Etienne, P.; Lequien, S.; Creuzet; G. J. Magn. Magn. Mater. 1991, 90, 95100.

27. Fullerton, E. E.; Kelly, D. M.; Guimpel, J.; Schuller, I. K.; Bruynseraede, Y. Phys. Rev. Lett. 1992, 68, 859-862.

28. Thomson, T.; Riedi, P. C.; Greig, D. Phys. Rev. B 1994, 50, 10319-10322.

29. Suzuki, M.; Taga, Y. Phys. Rev. B 1995, 52, 361-365.

30. Zhang, Z.; Lagally, M. G. Science 1997, 276, 377-383.

31. Camarero, J.; Graf, T.; de Miguel, J. J.; Miranda, R.; Kuch, W.; Zharnikov, M.; Dittschar, A.; Schneider, C. M.; Kirschner, J. Phys. Rev. Lett. 1996, 76, 4428-4431.

32. Tölkes, C.; David, R.; Tschersich, K. G.; Comsa,G.; Zeppenfeld, P. Europhys. Lett. 1999, 46, 589-594.

33. Zheng, M.; Shen, J.; Barthel, J.; Ohresser, P.; Mohan, Ch. V.; Kirschner, J. J. Physics: Condensed Matter to be published.

34. Padovani, S.; Scheurer, F.; Bucher, J.-P. Europhys. Lett. 1999, 45, 327-333.

35. Barbier, A.; Belkhou, R.; Ohresser, P.; Da Costa, V.; Guillot, C.; Carrière, B.; Deville, J.-P. Surface Science 1998, 414, 170186.

36. Scheurer, F.; Ohresser, P.; Bulou, H.; Deville, J.-P.; Carrière, B.; Dobroiu, A. Phys. Rev. B 1997, 56, 13490-13495.
37. Boukari, S.; Beaurepaire, E.; Bulou, H.; Carrière, B.; Deville, J.-P.; Scheurer, F.; Baudoing-Savois, R.; De Santis, M. Surface Science 1999, 430, 37-44.

38. Robinson, I. K.; Tweet, D. J. Rep. Prog. Phys. 1992, 55, 599651.

39. Barbier, A.; Renaud, G. Surface Science 1997, 392, L15-L18.

40. Stern, E. A.; Sayers,D. E. Lytle, F. W. Phys. Rev. B 1975, 11, 4836-4846.

41. Ohresser, P. Croissance, structure et magnétisme de l'interface cobalt-chrome; Thèse de doctorat en sciences, Université Louis Pasteur de Strasbourg, 1996.

42. Heckmann, O.; Magnan, H.; Le Fevre, P.; Chandesris, D.; Rehr, J. J. Surface Science 1994, 312, 62-72.

43. Stöhr, J. In: X-Ray Absorption. Principles, Applications, Techniques of EXAFS, SEXAFS and XANES (Koninsberger, D. C.; Prins, R. Eds); John Wiley \& Sons: New York, 1988, $443-$ 571.

44. Magnan, H.; Chandesris, D.; Villette, B.; Heckmann, O.; Lecante, J. Phys. Rev. Lett. 1991, 67, 859-862.

45. Rehr, J. J.; Albers, R. C. Phys. Rev. B 1990, 41, 8139-8149.

46. Le Fevre, P.; Magnan, H.; Heckmann, O.; Briois, V.; Chandesris, D. Phys. Rev. B 1995, 52, 11462-11466.

47. Pizzini, S.; Baudelet, F.; Chandesris, D.; Fontaine, A.; Magnan, H.; George, J. M.; Petroff, F.; Barthélémy, A.; Fert, A.; Loloe, R.; Schroeder, P. A. Phys. Rev. B 1992, 46, 12531256.

48. Panissod, P.; Jay, J. P.; Mény, C.; Wójcik, M.; Jedryka, E.; Hyperfine Int. 1996, 97-98, 75-98.

49. Dieny, B. J. Magn. Magn. Mater. 1994, 136, 335-359.

50. Lefakis, H.; Speriosu, V. S.; Gurney, B. A.; Humbert, P.; Benaissa, M.; Werckmann, J. J. Magn. Magn. Mater. 1996, 154, 17-23.

51. Bensmina, F. Magnétisme et structure de sandwiches à base de Co : Influence des recuits, Thèse de doctorat en sciences, Université Louis Pasteur de Strasbourg, 1999.

52. Azizi, A.; El Chahal, L.; Ounadjela, K.; Deville, J. P.; Thompson, S. M.; Gregg, J. F. J. Appl. Phys. 1996, 79, 62476249. 